\title{
Update from the Life Science X-ray Scattering (LiX) beamline at NSLS-II
}

\author{
Lin Yang
}

NSLS-II, Brookhaven National Laboratory, Upton, NY 11973, lyang@bnl.gov

We report the recent development of experimental capabilities at the LiX beamline. The LiX beamline was designed [1] to support x-ray scattering measurements on biological samples. The user operations started in late 2016. Since then, we have narrowed our research focus to solution scattering and microbeam structural mapping, and developed dedicated instrumentation optimized for these measurements. For solution scattering, a 6-axis robot is now used together with a temperaturecontrolled sample storage unit, with a capacity of 360 samples, and an automatic sample handler to perform automated static measurements. In-line liquid chromatography is integrated with beamline control software and the beamline can switch instantaneously between static and in-line measurements. All data are processed automatically immediately after the measurements. For microbeam mapping, we have implemented fly scans to support both 2D mapping and tomography. A Pilatus $900 \mathrm{~K}$ detector with an aperture in the sensing area was recently installed to improve the azimuthal angle coverage at wide scattering angles. The instrumentation that supports different experiments share the same mechanical interface to the support structure to enable rapid switching between experiments.

References:

[1] DiFabio et.al., AIP Conference Proceedings 1741, 030049 (2016) 